\title{
Role of cis-Monounsaturated Fatty Acids in the Prevention of Coronary Heart Disease
}

\author{
Peter J. Joris $^{1} \cdot$ Ronald P. Mensink ${ }^{1}$ \\ Published online: 24 May 2016 \\ (C) The Author(s) 2016. This article is published with open access at Springerlink.com
}

\begin{abstract}
The effects of cis-monounsaturated fatty acids (cisMUFAs) on the risk of coronary heart disease (CHD) and on CHD mortality are not clear. Also, dietary recommendations for cis-MUFA as derived by various organizations are not in agreement. Earlier studies have mainly focused on the effects of cis-MUFA on serum lipids and lipoproteins. More recent studies, however, have also addressed effects of cis-MUFA on other non-traditional CHD risk markers such as vascular function markers, postprandial vascular function, and energy intake and metabolism. Although well-designed randomized controlled trials with CHD events as endpoints are missing, several large prospective cohort studies have recently been published on the relationship between cis-MUFA and CHD risk. The aim of this paper is to review these new studies that have been published in the last 3 years on the effects of cisMUFA on cardiovascular risk markers and CHD.
\end{abstract}

Keywords Monounsaturated fatty acids $\cdot$ Oleic acid $\cdot$ Risk markers · Coronary heart disease $\cdot$ Cardiovascular disease

\section{Introduction}

Optimizing dietary fatty-acid intake is an integral part of dietary guidelines to prevent coronary heart disease (CHD) [1]. In fact, unequivocal evidence exists that eliminating trans-fatty

This article is part of the Topical Collection on Nutrition

Ronald P. Mensink

r.mensink@maastrichtuniversity.nl

1 Department of Human Biology, NUTRIM School of Nutrition and Translational Research in Metabolism, Maastricht University Medical Center, PO Box 616, Maastricht 6200 MD, The Netherlands acids from hydrogenated oils in the diet lowers the prevalence of CHD [2]. A decrease in the intake of saturated fatty acids (SFA) is also recommended, but in this respect, the type of macronutrient that replaces SFA is important. Replacement of SFA by carbohydrates from refined starches/added sugars may not decrease CHD risk, while replacement by carbohydrates from whole grains or cis-polyunsaturated fatty acid (cisPUFA) does [3•, 4]. The effects of cis-monounsaturated fatty acids (MUFA; Fig. 1) on CHD risk and CHD mortality are however less clear [5]. Also, dietary recommendations as derived by various health agencies for trans-fatty acids, SFA, and cis-PUFA are generally more in agreement than those for cis-MUFA. For example, no specific dietary reference values for $c i s$-MUFA have been formulated in the very recent 2015-2020 Dietary Guidelines for Americans [6], while other organizations have set reference values for cis-MUFA [7]. In 2012, Schwingshackl and Hoffmann have reviewed the available evidence from systemic reviews and meta-analyses regarding cis-MUFA intake and cardiovascular risk [8]. It was concluded that there was no clear justification to formulate specific dietary recommendations for cis-MUFA for the primary and secondary prevention of cardiovascular disease (CVD). On the other hand, as no harmful effects of cisMUFA-rich diets are known, more longer term intervention studies were suggested to clarify potential benefits of cisMUFA-rich diets. Since then, several new studies have been published during the last 3 years on the relationship between cis-MUFAs and cardiovascular risk markers or CHD, which will be discussed in the present review.

\section{Food Sources Of cis-Monounsaturated Fatty Acids}

Worldwide, the mean intake of cis-MUFA ranges from 3.5\% of total energy in certain regions of China to about $22 \%$ in 

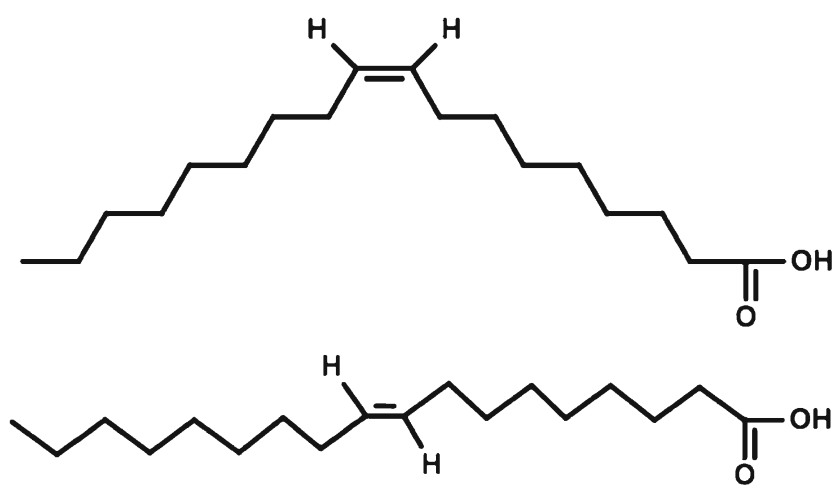

Fig. 1 Monounsaturated fatty acids (MUFAs) are chemically classified as fatty acids that have one double bond in the carbon chain. In the cisconfiguration, the hydrogen atoms attached to the double bond point into the same direction (top: oleic acid, a cis-MUFA with 18 carbon atoms), while in the trans-configuration, the hydrogen atoms are located on opposite sides (bottom: elaidic acid, a trans-MUFA with 18 carbon atoms)

Greece. Oleic acid $(18: 1 ; n-9)$ is the predominant $c i s$-MUFA, accounting for more than $92 \%$ of all MUFAs consumed. Other cis-MUFAs are also present in the diet, but only in very small amounts (Table 1). Except for erucic acid $(22: 1 ; n-9)$, their health effects have hardly been studied. Animal studies have suggested that erucic acid at high intakes may cause myocardial lipidosis due to poor mitochondrial $\beta$-oxidation. However, rapeseed oil varieties low in erucic acid are nowadays part of the food chain. Therefore, cis-MUFA intakes in the studies discussed mainly refer to intakes of oleic acid.

Some vegetable oils, such as olive oil $(\approx 75 \%)$, mid-oleic sunflower oil $(\approx 70 \%)$, and rapeseed oil $(\approx 65 \%)$, consist of

Table 1 Overview of different types of cis-monounsaturated fatty acids

\begin{tabular}{ll}
\hline cis-MUFA & Food sources \\
\hline Caproleic acid $(10: 1)$ & Ruminant fats \\
Lauroleic acid $(12: 1 ; n-3)$ & Ruminant fats \\
Myristoleic acid $(14: 1 ; n-5)$ & Ruminant fats \\
Palmitoleic acid $(16: 1 ; n-7)$ & Ruminant fats, fats from fish and \\
& marine mammals, macadamia oil, \\
& sea buckthorn oil and milkweed \\
& seed oil \\
Oleic acid $(18: 1 ; n-9)$ & Vegetable oils such as olive oil, \\
& mid-oleic sunflower oil and \\
& low-erucic acid rapeseed oil, nuts \\
& and seeds, avocados, palm oil, \\
& and animal fats \\
Gadoleic acid $(20: 1 ; n-11)$ & Fish oils such as ray, shark and \\
& cod, and mustard oil \\
Erucic acid $(22: 1 ; n-9)$ & Mustard oil \\
Nervonic acid $(24: 1 ; n-9)$ & Mustard oil, fish oils such as salmon
\end{tabular}

Oleic acid is the predominant cis-MUFA in the diet, accounting for more than $92 \%$ of all MUFAs consumed

MUFA monounsaturated fatty acid more than $50 \%$ of $c i s$-MUFA, followed by other products such as palm oil, nuts and seeds, avocados, and animal fats [9]. In fact, animal fats are the main source of cis-MUFA in a typical American diet, and high cis-MUFA and SFA intakes may thus be correlated. The six food groups with the highest contribution to total MUFA intake among US adults based on the National Health and Nutrition Examination Survey (NHANES) 2003-2006 were other fats and oils (10.5\%), beef (9.2\%), cakes/cookies/quick bread/pastry/pie (8.9\%), frankfurters/sausages/luncheon meats $(7.5 \%)$, cheese $(6.6 \%)$, and margarine and butter $(6.4 \%)$ [10].

\section{cis-Monounsaturated Fatty Acid and Cardiovascular Risk Markers}

cis-MUFA may affect cardiovascular health through effects on a wide variety of markers associated with CHD [11]. This paragraph reviews current evidence on the effects of cisMUFA on serum lipids and lipoproteins, vascular function markers, postprandial vascular function, and energy intake and metabolism.

\section{Serum Lipids and Lipoproteins}

Data from the many earlier well-controlled randomized intervention studies have shown that cis-MUFA has a favorable effect on the serum lipoprotein profile. In studies that replaced fats or oils high in SFA with oils rich in cis-MUFA, significant decreases were found in serum concentrations of total cholesterol, LDL-cholesterol, and apoB100 and the total to HDLcholesterol ratio. Serum HDL-cholesterol, apo-AI, and triacylglycerol concentrations hardly changed. Overall, the effects of oils rich in cis-PUFA were slightly more favorable than those of oils rich in cis-MUFA, especially on LDLcholesterol and the total to HDL-cholesterol ratio. In these studies, the diets were enriched with cis-MUFA from different sources such as olive oil, high-oleic acid sunflower oil, higholeic acid safflower oil, and rapeseed oil. More recently, other intervention trials have been carried out specifically focusing on cis-MUFA. Gilmore et al. reported that in 17 postmenopausal women, consumption of high-MUFA or low-MUFA ground beef for 6 weeks had comparable effects on the serum lipoprotein profile [12]. However, the difference in cis-MUFA intake provided by the two types of ground beef was less than $2 \mathrm{~g}$ /day, which may have been too small to observe any effects. In a randomized, double-blind, crossover design with 131 abdominally obese volunteers, five oils with comparable amounts of SFA, but differing in the amounts of oleic acid, linoleic acid, $\alpha$-linolenic acid, or docosahexaenoic acid (DHA) were consumed for 4-week periods [13••]. Effects on serum LDL-cholesterol, HDL-cholesterol, and triacylglycerol concentrations were comparable, confirming the findings that 
cis-unsaturated fatty acids with 18 carbon atoms have comparable effects on serum lipids. An oil rich in cis-MUFA and DHA, however, increased LDL-cholesterol and HDLcholesterol and lowered serum triacylglycerol concentrations as compared with the other four oils [13••]. In a subset of the population, no evidence was found that consumption of the oils had an adverse effect on LDL proteoglycan binding [14], as observed in animal studies after consumption of oils rich in cis-MUFA [15]. As expected, a high-palmitic acid diet increased concentrations of total LDL-cholesterol and HDLcholesterol and had no effects on serum triacylglycerol as compared with a high-oleic acid diet in 18 healthy subjects [16]. Also, olive oil and rapeseed oil were found to have similar effects on the serum lipoprotein profile [17]. In a 24week parallel study in individuals with type 2 diabetes, a peanut-enriched American Diabetic Association (ADA) meal plan had similar effects on the serum lipoprotein profile compared with a nut-free ADA meal plan [18]. Dietary SFA and total fat intakes between the two groups were not different. In the Dietary Intervention and Vascular Function (DIVAS) trial, a randomized, single-blind, parallel-group intervention study with 195 adults at moderate CVD risk, three groups of subjects consumed for 16 weeks a diet that was rich in SFA, cisMUFA, or cis-PUFA. Except for commercially available foods, specially formulated oils and spreads were used high in cis-MUFA (refined olive oil and rapeseed oil) and cisPUFA (safflower oil). The high-SFA group received butter. As compared with the high-SFA groups, serum total and LDL-cholesterol concentrations were reduced to the same extent on the high-MUFA and high-PUFA diets. Effects on serum HDL-cholesterol and triacylglycerol were comparable [19••]. Overall, these recent studies are in line with the earlier studies.

The mechanistic aspects of cis-MUFA were examined by Labonté and colleagues [20]. In a randomized parallel study, the effects of exchanging carbohydrates for cis-MUFA as part of an experimental portfolio diet on apolipoprotein kinetics were investigated in 16 dyslipidemic subjects. The experimental diets were fed for 4 weeks after a 4 -week run-in period. The high-MUFA diet increased apo-AI pool size, mainly due to a reduced apo-AI fractional catabolic rate. LDL apoB100 pool size tended to be reduced on the cis-MUFA diet through an increase in LDL apoB100 fractional catabolic rate.

\section{Vascular Function Markers}

The DIVAS study was adequately powered to investigate the long-term impact of replacing SFAs with cis-MUFAs on various fasting vascular function markers [19••]. It was found that replacing dietary SFAs with cis-MUFAs did not affect fasting flow-mediated vasodilation (FMD) of the brachial artery $[19 \bullet \bullet$. These findings are in agreement with those of Sanders and colleagues, who replaced $5.2 \%$ of energy from dietary SFAs by cis-MUFAs for 24 weeks in 121 insulinresistant men and women [21••]. Furthermore, replacement of SFAs had no effect on arterial stiffness, supporting the earlier findings of Sanders et al. reporting no change in carotid-to-femoral pulse wave velocity $\left(\mathrm{PWV}_{\mathrm{c}-\mathrm{f}}\right)$ when SFAs were replaced with cis-MUFAs [21••]. Of the secondary outcome measures, substitution of dietary SFAs by cis-MUFAs significantly reduced circulating E-selectin concentrations by $7.8 \%$, suggesting an improvement in endothelial activity, while night systolic blood pressure was reduced by $4.9 \mathrm{~mm}$ of $\mathrm{Hg}$. As discussed by the authors [19••], the large range of recorded daily activity levels may have masked any effects of the intervention on 24-h or daytime ambulatory blood pressure recordings.

\section{Postprandial Vascular Function}

Postprandial vascular responses were compared between normal-weight and obese men following three isocaloric high-fat challenges differing in fatty-acid composition [22]. For this, 18 normal-weight and 18 obese middle-aged men received in a random order a milkshake providing $95 \mathrm{~g}$ of fat, which was either high in SFAs, cis-MUFAs, or $n$-3 longchain PUFAs. Compared with the SFA and $n-3$ PUFA shakes, it was found that the cis-MUFA milkshake resulted in a more pronounced decrease in the augmentation index (AIx) - a measure of the arterial pressure waveform that depends on the tone of peripheral resistance arteries - and blood pressure. Milkshake consumption resulted in increased plasma sICAM1, sICAM3, and sVCAM1 concentrations $4 \mathrm{~h}$ postprandially, with no differences in responses between the shakes for these and other (E-selectin and vWF) plasma biomarkers of endothelial function. Lithander et al. [23] compared the effects of a test meal rich in oleic acid with an isoenergetic meal rich in palmitic acid on postprandial vascular function in younger male participants. No differences in PWV $_{\text {c-f }}$, AIx or blood pressure were found between the two test meals providing $56 \mathrm{~g}$ of fat. Except for subject characteristics, the difference in findings with the study of Esser and colleagues [22] may also be explained by meal composition, because the mixed meal provided by Lithander was higher in carbohydrates and lower in dietary fat (56 g of fat versus $95 \mathrm{~g}$ of fat).

\section{Energy Intake and Metabolism}

Mennella and colleagues fed 15 healthy normal-weight subjects in random order $30 \mathrm{~mL}$ of high-oleic acid sunflower oil, virgin olive oil, or sunflower oil plus $30 \mathrm{~g}$ of bread. After consumption of the oils rich in oleic acid, energy intake was reduced at the subsequent self-chosen lunch, possible related to the increased postprandial concentrations of oleoylethanolamide (OEA), a compound produced by the small 
intestine that is involved in appetite regulation. However, energy intake over the next $24 \mathrm{~h}$ as assessed by food diaries was comparable between the three treatments [24]. Total energy intake as measured during 4 days was lower in a randomized controlled trial (RCT) with 24 healthy elderly overweight participants following consumption of high-oleic acid peanuts and regular peanuts compared with isoenergetic amounts of a highcarbohydrate snack (potato crisps). Despite these reductions in energy intake, no differences in hunger and satiety that were assessed following snack consumption using visual analog scales were observed [25]. Comparable results were reported in another study with high-oleic acid peanuts [26].

In their recent review [27], Krishnan and Cooper concluded that acute-meal studies suggested that diet-induced thermogenesis and fat oxidation were increased on high unsaturatedfat diets as compared with SFA-rich diets. In this respect, no differences were found between MUFA and PUFA. It was further concluded that also long-term dietary interventions may suggest that MUFA-rich diets induced a greater energy expenditure, diet-induced thermogenesis or fat oxidation as compared with SFA-rich diets [27]. More recently, however, Clevenger et al. found no differences in the effects of SFA, MUFA, and PUFA on diet-induced thermogenesis or postprandial substrate oxidation in obese women [28]. On the other hand, Kien and colleagues reported in healthy volunteers a higher rate of fat oxidation during the fasted state on a palmitic-acid rich diet as compared with an oleic-acid rich diet. Diets were provided for 3 weeks [29••]. Overall, there is no unanimous agreement that cis-MUFA affects energy metabolism compared with other dietary fatty acids. The few short-term studies that used peanuts are more consistent in this respect and effects may therefore not necessarily be related to cis-MUFA intake. Also, it is not known if the effects are sustained on the longer term and are ultimately translated into a lower body weight and an improved cardiometabolic profile.

\section{cis-Monounsaturated Fatty Acid and Cardiovascular Disease}

The Mediterranean diet is well known for its high cisMUFA content (16 to $29 \%$ of energy) with olive oil being the predominant source of fat and intakes of SFA below $8 \%$ [30]. The Mediterranean dietary pattern is associated with a reduced cardiovascular risk. Even though not designed to specially evaluate the effects of cis-MUFA, the PREvención con DIEeta MEDiterraneá (PREDIMED) trial found that a Mediterranean diet supplemented with extravirgin olive oil $(50 \mathrm{~g} /$ day $)$ or mixed nuts $(30 \mathrm{~g} /$ day $)$ reduced in individuals at high cardiovascular risk the incidence of major cardiovascular events (a composite of myocardial infarction, stroke, or death from CVD causes) as compared with a control diet low in fat [31••]. A meta-analysis of 15
RCTs, that involved more than 50,000 participants, aimed to estimate the effect of replacing dietary SFA for carbohydrates, cis-PUFA, cis-MUFA, or protein on cardiovascular morbidity and mortality [5]. It was concluded that the effects of cis-MUFA were unclear as only one small trial from 1965 was identified [32], in which no effects were observed. In this respect, the results of recently published large prospective cohort studies evaluating the association between cisMUFA intake with the risk of CVD and cardiovascular death may be more informative (Table 2).

Subjects within the PREDIMED trial were also prospectively studied. While the trial was conducted from 2003 to 2010, the present data were based on an expanded follow-up until 2012 [33•]. The mean intake of cis-MUFAs (expressed as percentage of energy) was high: $13.4 \%$ in the lowest quintile compared with $26.1 \%$ in the top quintile. After 6 years of follow-up, $336 \mathrm{CVD}$ cases and 414 total deaths were reported. It was found that higher intakes of cis-MUFA were inversely associated with CVD, cardiovascular death, and all-cause death. In fact, isocaloric substitution of $5 \%$ of energy from SFAs or trans-FAs with cis-MUFAs was associated with a 37 and $40 \%$ lower risk of total CVD events. In agreement, an updated analysis of the Nurses' Health Study (1980-2010; 84, 628 US women) and the Health Professional Follow-Up Study (1986-2010; 42,908 US men) showed a reduction in CHD risk when SFA was exchanged for cis-MUFA [3•]. In these two large, independent prospective cohorts of men and women, 7667 cases of CHD (4931 nonfatal myocardial infarctions and 2736 CHD deaths) were documented over 24 to 30 years of follow-up. Replacing $5 \%$ of energy from dietary SFA with an equivalent amount of cis-MUFA was associated with a $15 \%(95 \%$ CI, 3 to $26 \%)$ lower risk of CHD. In a large prospective cohort study carried out within the AlphaTocopherol, Beta-Carotene Cancer Prevention Study (ATBC), the associations between glycemic index, substitutions of total, low-, medium-, and high-glycemic-index carbohydrates for dietary fat and the risk of CHD were examined [34•]. During a 19-year follow-up, 4379 CHD cases, including a total of 2377 non-fatal myocardial infarctions and 2002 CHD deaths, were documented among approximately 22, 000 middle-aged Finnish male smokers. Substituting carbohydrates for $c i s$-MUFAs was associated with a decreased risk. It was estimated that isoenergetic replacements of $2 \%$ of energy from total, low-, or high-glycemic-index carbohydrates for cis-MUFAs were associated with $8 \%(95 \%$ CI, 1 to $16 \%$ ), $9 \%$ (95\% CI 2 to $16 \%$ ), and $8 \%$ (95\% CI 1 to $15 \%)$ lower CHD risks. The isocaloric replacement of medium-glycemic-index carbohydrates with cis-MUFA tended to decrease the risk of CHD by $7 \%$ (95\% CI 0 to $15 \%$ ). Dietary fiber intake modified the association between the replacement of carbohydrates with cis-MUFA and the risk of CHD in the stratified analyses. In fact, increasing cisMUFA intake at the expense of carbohydrates was more 
Table 2 Summary of recent studies assessing the effects of cis-MUFAs on cardiovascular disease

\begin{tabular}{|c|c|c|c|}
\hline Study & Research question & Study design & Results \\
\hline PREDIMED Study [33•] & $\begin{array}{l}\text { Dietary fat intake and risk of total } \\
\text { CVD events in a population at } \\
\text { high risk of CVD }\end{array}$ & Prospective cohort study & $\begin{array}{l}\text { Dietary SFA for } c i s-\text { MUFA } \\
\text { (5\% of energy) HR } 0.63 \\
\text { (95\% CI } 0.43-0.94)\end{array}$ \\
\hline NHS and the HPFS [3•] & $\begin{array}{l}\text { Dietary fat intake and risk of CHD } \\
\text { in men and women free of } \\
\text { diabetes, CVD, and cancer }\end{array}$ & Prospective cohort study & $\begin{array}{l}\text { Dietary SFA for } c i s-\text { MUFA } \\
\text { (5\% of energy) HR } 0.85 \\
\text { (95\% CI } 0.74-0.97)\end{array}$ \\
\hline $\begin{array}{l}\text { ATBC Cancer Prevention } \\
\text { Study }[34 \bullet]\end{array}$ & $\begin{array}{l}\text { Carbohydrate substitution for } \\
\text { dietary fat and risk of CHD in } \\
\text { Finnish male smokers }\end{array}$ & Prospective cohort study & $\begin{array}{l}\text { Total carbohydrates for cis- } \\
\text { MUFA (2\% of energy) } \\
\text { RR } 0.92(95 \% \text { CI } 0.84-0.99)\end{array}$ \\
\hline EPIC Study [35•] & $\begin{array}{l}\text { Carbohydrate substitution for } \\
\text { dietary fat on mortality risk in } \\
\text { patients with type } 2 \text { diabetes }\end{array}$ & Prospective cohort study & $\begin{array}{l}\text { Carbohydrates for cis-MUFA } \\
\text { (5\% of energy) HR } 0.87 \\
\text { (95\% CI 0.76-1.00). No } \\
\text { effects on CVD mortality risk }\end{array}$ \\
\hline EPIC-NL Study [36•] & $\begin{array}{l}\text { Dietary fat intake and risk of } \\
\text { CHD in a Dutch population }\end{array}$ & Prospective cohort study & $\begin{array}{l}\text { Dietary SFA for } c i s-M U F A \\
\text { (5\% of energy) HR } 1.30 \\
\text { (95\% CI } 1.02-1.65)\end{array}$ \\
\hline
\end{tabular}

CVD cardiovascular disease, $C H D$ coronary heart disease, $N / A$ not applicable, $S F A$ saturated fatty acid, $M U F A$ monounsaturated fatty acid, $H R$ hazards ratio, $R R$ relative risk

beneficial among the subjects with a higher fiber intake. In patients with type 2 diabetes, the association between dietary carbohydrate intake and substitution for cis-MUFA with cardiovascular and all-cause mortality was also investigated [35•]. A total of 6192 type 2 diabetic patients from 15 cohorts of the European Prospective Investigation into Cancer and Nutrition (EPIC) were included. After a mean follow-up of about 9 years, 268 CVD deaths and 791 total deaths were reported. Substituting $5 \%$ of energy from carbohydrates with cis-MUFA may be associated with a lower all-cause mortality risk (HR, $0.87 ; 95 \% \mathrm{CI}, 0.76$ to 1.00 ), while no effects were reported for CVD mortality risk.

Surprisingly, a lower CHD risk with a higher intake of SFAs was recently observed that did not depend on the type of substituting macronutrient [36•]. The EPICNetherlands (EPIC-NL) cohort included 35,597 Dutch men and women. In this prospective cohort study, a total of 1807 incident CHD cases and 158 CHD deaths (8.7\%) occurred over a median follow-up time of 12 years. After full adjustment, the substitution of $5 \%$ of energy from SFA with cis-MUFA was associated with $30 \%$ (95\% CI: $2 \%$ to $65 \%$ ) increased risk to develop CHD. These results, however, should be interpreted with caution. As discussed by the authors [36•], residual confounding by unmeasured initiation of cholesterol-lowering therapy during follow-up may explain these findings as adults with high SFA intake have high serum cholesterol concentrations and will become eligible for lipid-lowering therapy during follow-up, which would reduce CHD risk. In addition, the limited variation in dietary SFA intake or the source of SFA may also have attributed to the observed risk differences in this Dutch population.

\section{Conclusions}

In summary, recent studies are in line with the earlier studies showing that $c i s$-MUFAs have a favorable effect on the serum lipoprotein profile as compared with a mixture of SFA, while effects are comparable to those of linoleic and $\alpha$-linolenic acid. Effects on fasting and postprandial vascular function have not been studied extensively and no consistent differences between the various fatty acids are evident. Longer-term studies should address whether products rich in cis-MUFA affect energy intake and metabolism compared with other macronutrients. In fact, studies addressing the effects of food sources and matrices are of interest, as this may impact the results. Well-designed RCTs with CVD events as endpoints are lacking. Evidence from large prospective cohort studies regarding effects of $c i s$-MUFA on the risk to develop CHD is limited, but several studies do suggest that replacements of SFA or high-glycemic index foods for cisMUFA lowers CHD risk. In this respect, however, cis-MUFA is not more beneficial than linoleic acid. More research is thus required on the long-term effects of cis-MUFA as compared with other macronutrients on CHD risk markers as well as on clinical endpoints to clarify the potential role of cis-MUFAs in the primary and secondary prevention of CHD.

\section{Compliance with Ethical Standards}

Conflict of Interest Peter J. Joris declares no conflict of interest.

Ronald P. Mensink declares grant support from Top Institute for Food and Nutrition (Wageningen, the Netherlands) and from Unilever Research and Development (Vlaardingen, the Netherlands).

Human and Animal Rights and Informed Consent This article does not contain any studies with human or animal subjects performed by any of the authors. 
Open Access This article is distributed under the terms of the Creative Commons Attribution 4.0 International License (http:// creativecommons.org/licenses/by/4.0/), which permits unrestricted use, distribution, and reproduction in any medium, provided you give appropriate credit to the original author(s) and the source, provide a link to the Creative Commons license, and indicate if changes were made.

\section{References}

Papers of particular interest, published recently, have been highlighted as:

- Of importance

-• Of major importance

1. Lichtenstein AH, Appel LJ, Brands M, Carnethon M, Daniels S, Franch HA, et al. Diet and lifestyle recommendations revision 2006: a scientific statement from the American Heart Association Nutrition Committee. Circulation. 2006;114:82-96.

2. Mozaffarian D, Clarke R. Quantitative effects on cardiovascular risk factors and coronary heart disease risk of replacing partially hydrogenated vegetable oils with other fats and oils. Eur J Clin Nutr. 2009;63:S22-33.

3. Li Y, Hruby A, Bernstein AM, Ley SH, Wang DD, Chiuve SE, et al. Saturated fats compared with unsaturated fats and sources of carbohydrates in relation to risk of coronary heart disease: a prospective cohort study. J Am Coll Cardiol. 2015;66:1538-48. This prospective cohort study suggests that replacement of saturated fatty acids for unsaturated fatty acids and/or high-quality carbohydrates reduces $\mathrm{CHD}$ risk.

4. Mozaffarian D, Micha R, Wallace S. Effects on coronary heart disease of increasing polyunsaturated fat in place of saturated fat: a systematic review and meta-analysis of randomized controlled trials. PLoS Med. 2010;7, e1000252.

5. Hooper L, Martin N, Abdelhamid A, Davey Smith G. Reduction in saturated fat intake for cardiovascular disease. Cochrane Database Syst Rev. 2015;6, CD011737.

6. U.S. Department of Health and Human Services and U.S. Department of Agriculture. 2015-2020 Dietary Guidelines for Americans. 8th Edition. December 2015. Available at: http:// health.gov/dietaryguidelines/2015/guidelines/. Accessed on March 3, 2016.

7. Aranceta J, Perez-Rodrigo C. Recommended dietary reference intakes, nutritional goals and dietary guidelines for fat and fatty acids: a systematic review. Br J Nutr. 2012;107:S8-22.

8. Schwingshack1 L, Hoffmann G. Monounsaturated fatty acids and risk of cardiovascular disease: synopsis of the evidence available from systematic reviews and meta-analyses. Nutrients. 2012;4: 1989-2007.

9. Degirolamo C, Rudel LL. Dietary monounsaturated fatty acids appear not to provide cardioprotection. Curr Atheroscler Rep. 2010;12:391-6.

10. O'Neil CE, Keast DR, Fulgoni VL, Nicklas TA. Food sources of energy and nutrients among adults in the US: NHANES 20032006. Nutrients. 2012;4:2097-120.

11. Hammad S, Pu S, Jones PJ. Current evidence supporting the link between dietary fatty acids and cardiovascular disease. Lipids. doi: 10.1007/s11745-015-4113-x.

12. Gilmore LA, Crouse SF, Carbuhn A, Klooster J, Calles JA, Meade $\mathrm{T}$, et al. Exercise attenuates the increase in plasma monounsaturated fatty acids and high-density lipoprotein cholesterol but not highdensity lipoprotein $2 \mathrm{~b}$ cholesterol caused by high-oleic ground beef in women. Nutr Res. 2013;33:1003-11.
13.• Jones PJ, Senanayake VK, Pu S, Jenkins DJ, Connelly PW, Lamarche B, et al. DHA-enriched high-oleic acid canola oil improves lipid profile and lowers predicted cardiovascular disease risk in the canola oil multicenter randomized controlled trial. Am J Clin Nutr. 2014;100:88-97. This well-controlled study shows that oils rich in unsaturated fatty acids with 18 carbon atoms have comparable effects on the serum lipoprotein profile.

14. Jones PJ, MacKay DS, Senanayake VK, Pu S, Jenkins DJ, Connelly PW, et al. High-oleic canola oil consumption enriches LDL particle cholesteryl oleate content and reduces LDL proteoglycan binding in humans. Atherosclerosis. 2015;238:231-8.

15. Melchior JT, Sawyer JK, Kelley KL, Shah R, Wilson MD, Hantgan $\mathrm{RR}$, et al. LDL particle core enrichment in cholesteryl oleate increases proteoglycan binding and promotes atherosclerosis. J Lipid Res. 2013;54:2495-503.

16. Kien CL, Bunn JY, Stevens R, Bain J, Ikayeva O, Crain K, et al. Dietary intake of palmitate and oleate has broad impact on systemic and tissue lipid profiles in humans. Am J Clin Nutr. 2014;99:436-45.

17. Kruse M, von Loeffelholz C, Hoffmann D, Pohlmann A, Seltmann $\mathrm{AC}$, Osterhoff M, et al. Dietary rapeseed/canola-oil supplementation reduces serum lipids and liver enzymes and alters postprandial inflammatory responses in adipose tissue compared to olive-oil supplementation in obese men. Mol Nutr Food Res. 2015;59: 507-19.

18. Wien M, Oda K, Sabate J. A randomized controlled trial to evaluate the effect of incorporating peanuts into an American Diabetes Association meal plan on the nutrient profile of the total diet and cardiometabolic parameters of adults with type 2 diabetes. Nutr J. 2014;13:10.

19.• Vafeiadou K, Weech M, Altowaijri H, Todd S, Yaqoob P, Jackson $\mathrm{KG}$, et al. Replacement of saturated with unsaturated fats had no impact on vascular function but beneficial effects on lipid biomarkers, E-selectin, and blood pressure: results from the randomized, controlled Dietary Intervention and VAScular function (DIVAS) study. Am J Clin Nutr. 2015;102:40-8. This is a large randomized controlled trial reporting that replacement of saturated with unsaturated fats has no impact on vascular function.

20. Labonte ME, Jenkins DJ, Lewis GF, Chiavaroli L, Wong JM, Kendall CW, et al. Adding MUFA to a dietary portfolio of cholesterol-lowering foods reduces apoAI fractional catabolic rate in subjects with dyslipidaemia. Br J Nutr. 2013;110:426-36.

21.• Sanders TA, Lewis FJ, Goff LM, Chowienczyk PJ, Group RS. SFAs do not impair endothelial function and arterial stiffness. Am J Clin Nutr. 2013;98:677-83. This well-controlled trial shows in a large group of healthy individuals that replacement of saturated fatty acid with cis-monounstatured fatty acid does not affect vascular function.

22. Esser D, van Dijk SJ, Oosterink E, Muller M, Afman LA. A highfat SFA, MUFA, or n3 PUFA challenge affects the vascular response and initiates an activated state of cellular adherence in lean and obese middle-aged men. J Nutr. 2013;143:843-51.

23. Lithander FE, Herlihy LK, Walsh DM, Burke E, Crowley V, Mahmud A. Postprandial effect of dietary fat quantity and quality on arterial stiffness and wave reflection: a randomised controlled trial. Nutr J. 2013;12:93.

24. Mennella I, Savarese M, Ferracane R, Sacchi R, Vitaglione P. Oleic acid content of a meal promotes oleoylethanolamide response and reduces subsequent energy intake in humans. Food Funct. 2015;6: 204-10.

25. Barbour JA, Howe PR, Buckley JD, Wright GC, Bryan J, Coates AM. Lower energy intake following consumption of Hi-oleic and regular peanuts compared with iso-energetic consumption of potato crisps. Appetite. 2014;82:124-30.

26. Duarte Moreira Alves R, Boroni Moreira AP, Silva Macedo V, Brunoro Costa NM, Goncalves Alfenas Rde C, Bressan J. High- 
oleic peanuts increase diet-induced thermogenesis in overweight and obese men. Nutr Hosp. 2014;29:1024-32.

27. Krishnan S, Cooper JA. Effect of dietary fatty acid composition on substrate utilization and body weight maintenance in humans. Eur J Nutr. 2014;53:691-710.

28. Clevenger HC, Stevenson JL, Cooper JA. Metabolic responses to dietary fatty acids in obese women. Physiol Behav. 2015;139:73-9.

29.• Kien CL, Bunn JY, Tompkins CL, Dumas JA, Crain KI, Ebenstein DB, et al. Substituting dietary monounsaturated fat for saturated fat is associated with increased daily physical activity and resting energy expenditure and with changes in mood. Am J Clin Nutr. 2013;97:689-97. In this article, Kien and colleagues demonstrate that substituting monounsaturated fat for saturated fat is associated with an increased resting energy expenditure.

30. Gillingham LG, Harris-Janz S, Jones PJ. Dietary monounsaturated fatty acids are protective against metabolic syndrome and cardiovascular disease risk factors. Lipids. 2011;46:209-28.

31.• Estruch R, Ros E, Salas-Salvado J, Covas MI, Corella D, Aros F, et al. Primary prevention of cardiovascular disease with a Mediterranean diet. N Engl J Med. 2013;368:1279-90. The PREDIMED study is the first large randomized controlled trial showing the benefit of a Mediterranean diet pattern on CVD risk.

32. Rose GA, Thomson WB, Williams RT. Corn oil in treatment of ischaemic heart disease. Br Med J. 1965;1:1531-3.

33. Guasch-Ferre M, Babio N, Martinez-Gonzalez MA, Corella D, Ros E, Martin-Pelaez S, et al. Dietary fat intake and risk of cardiovascular disease and all-cause mortality in a population at high risk of cardiovascular disease. Am J Clin Nutr. 2015;102: 1563-73. This recently published prospective cohort study found that intakes of monounsaturated fatty acids are associated with a lower risk of CVD and death.

34. Simila ME, Kontto JP, Mannisto S, Valsta LM, Virtamo J. Glycaemic index, carbohydrate substitution for fat and risk of CHD in men. Br J Nutr. 2013;110:1704-11. Within the AlphaTocopherol, Beta-Carotene Cancer Prevention Study, a prospective cohort study in male smokers, replacements of carbohydrates for cis-MUFA reduced the risk of CHD.

35. Campmans-Kuijpers MJ, Sluijs I, Nothlings U, Freisling H, Overvad $\mathrm{K}$, Boeing $\mathrm{H}$, et al. The association of substituting carbohydrates with total fat and different types of fatty acids with mortality and weight change among diabetes patients. Clin Nutr. 2015. doi:10.1016/j.clnu.2015.08.003. In patients with type 2 diabetes, substitution of carbohydrates with MUFAs may be associated with lower CVD mortality.

36. Praagman J, Beulens JW, Alssema M, Zock PL, Wanders AJ, Sluijs I, et al. The association between dietary saturated fatty acids and ischemic heart disease depends on the type and source of fatty acid in the European Prospective Investigation into Cancer and NutritionNetherlands cohort. Am J Clin Nutr. 2016;103:356-65. Replacement of saturated fatty acids for other macronutrients was associated with a lower risk of CHD in this Dutch cohort from the European Prospective Investigation into Cancer and Nutrition. 